\title{
A new locus for autosomal dominant Charcot-Marie- Tooth disease type 2 (CMT2F) maps to chromosome 7q11-q21
}

\author{
Shoukhrat M Ismailov ${ }^{1}$, Valeriy P Fedotov ${ }^{3}$, Elena L Dadali ${ }^{1,2}$, Alexander V Polyakov ${ }^{2}$, \\ Christine Van Broeckhoven ${ }^{4}$, Vladimir I Ivanov ${ }^{1,2}$, Peter De Jonghe ${ }^{4,5}$, \\ Vincent Timmerman*,4 and Oleg V Evgrafov*,2,4
}

\begin{abstract}
${ }^{1}$ Russian State Medical University, Moscow, Russia; ${ }^{2}$ Research Center for Medical Genetics, Moscow, Russia; ${ }^{3}$ Genetic Counselling Department of Diagnostic Center, Voronezh, Russia; ${ }^{4}$ Laboratory of Molecular Genetics, Flanders Interuniversity Institute for Biotechnology (VIB), Born-Bunge Foundation (BBS), University of Antwerpen (UIA), Antwerpen, Belgium; ${ }^{5}$ Division of Neurology, University Hospital of Antwerpen (UZA), Antwerpen, Belgium
\end{abstract}

Charcot-Marie-Tooth disease (CMT) constitutes a genetically heterogeneous group of inherited motor and sensory peripheral neuropathies. The axonal type of CMT is designated CMT type 2 (CMT2). Four loci for autosomal dominant CMT2 have been reported so far. Only in CMT2E, linked to chromosome 8p21, diseasecausing mutations in the gene for neurofilament light chain (NEFL) were identified. In this study we report a multigenerational Russian family with autosomal dominant CMT2 and assign the locus to chromosome 7q11-q21. The CMT2 neuropathy in this family represents a novel genetic entity designated CMT2F. European Journal of Human Genetics (2001) 9, 646-650.

Keywords: Charcot-Marie-Tooth neuropathy; genome-wide search; genetic linkage

\section{Introduction}

Charcot-Marie-Tooth disease (CMT) is the most common inherited disorder of the peripheral nervous system. The prevalence of CMT is estimated as 10-40/100 000. Clinically CMT is characterised by progressive symmetrical weakness and atrophy of distal limb muscles, initially involving the legs and particularly the peroneal muscles, depressed or absent tendon reflexes, and mild to moderate distal sensory abnormalities. CMT disease can be divided into demyelinating and axonal forms on the basis of electrophysiological and histopathological criteria. CMT is genetically heterogeneous, and usually inherited as an autosomal dominant trait although all modes of inheritance have been described. ${ }^{1}$

*Correspondence: Prof Dr OV Evgrafov and Dr V Timmerman, Molecular Genetics Laboratory, Biochemistry Department, Universitaire Instelling Antwerpen (UIA), Universiteitsplein 1, B-2610 Antwerpen, Belgium. Tel: +32 382024 99; Fax: +323820 2541 ;

E-mail: evgrafov@hotmail.com and timmerm@uia.ua.ac.be Received 27 February 2001; revised 3 May 2001; accepted 9 May 2001
CMT type 2 (CMT2) represents the axonal type of CMT. In total seven loci for CMT2 have been reported (reviewed in ${ }^{2}$ ). Only in CMT2E, linked to chromosome $8 \mathrm{p} 21$, diseasecausing mutations in the gene for neurofilament light chain (NEFL) were identified. ${ }^{3,4}$ We report a Russian autosomal dominant CMT2 family that shows linkage to a novel locus on 7q11-q21. This finding suggests that the CMT2 neuropathy in this family represents a novel genetic entity designated CMT2F.

\section{Materials and methods}

Family

We studied a single large six-generation family with autosomal dominant CMT2 from the Voronezh province of Russia (Figure 1). Informed consent was obtained from all family members who participated in the study. During the last 2 years, 36 members of the family were clinically investigated and the majority of them were also electrophysiologically examined. Fourteen individuals were diag- 


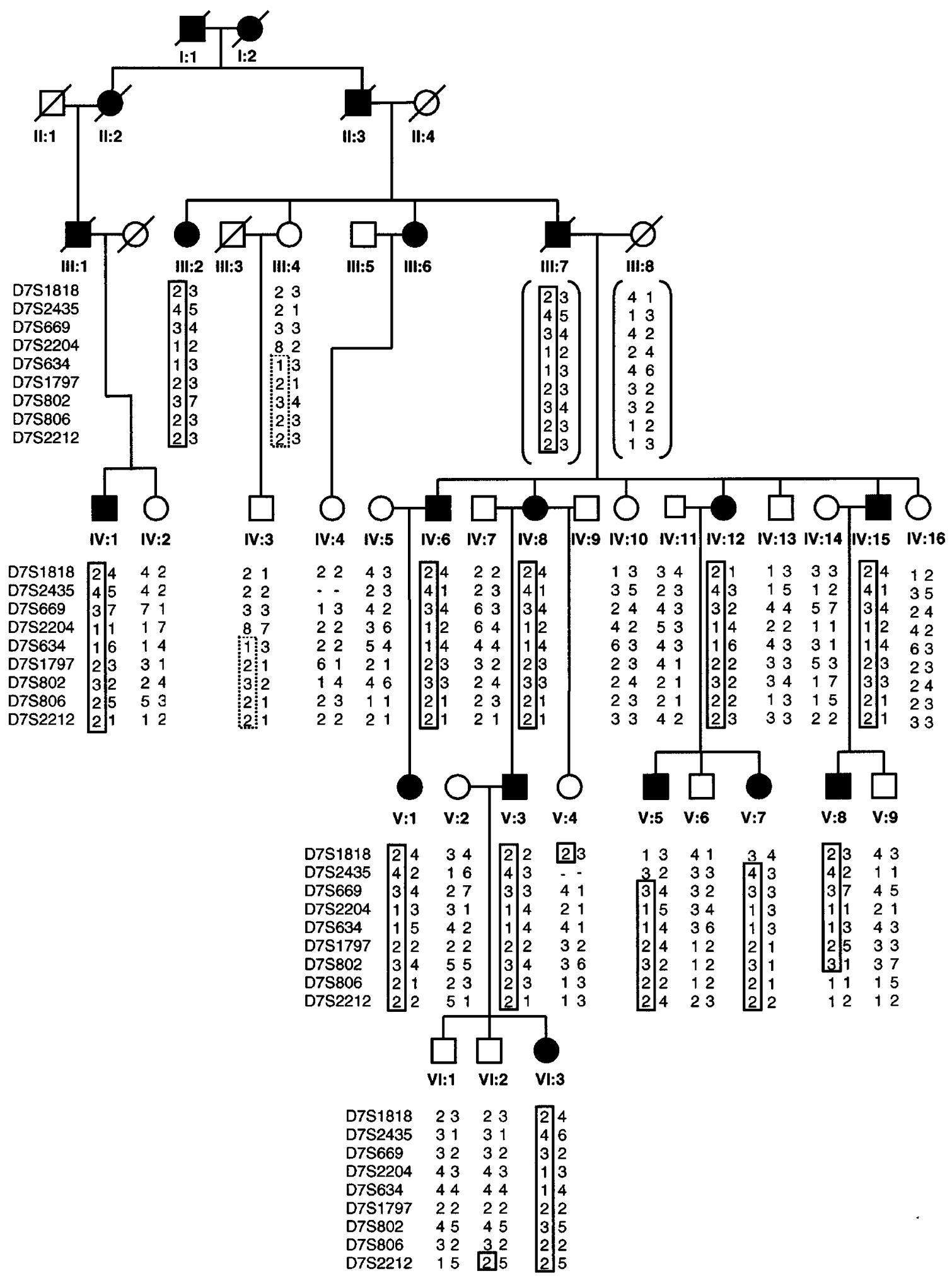

Figure 1 Pedigree and haplotypes of the Russian CMT2 family. Only family members included in the genetic linkage analysis are shown. Filled squares and circles indicate affected males and females respectively. Open squares and circles represent unaffected males and females respectively. The disease associated haplotype is boxed, the haplotype in a dotted box for person III. 4 is discussed in the text, the haplotypes between brackets are deduced. The boxed haplotype $-=$ no data. 
nosed as CMT2. All affected individuals have a similar clinical phenotype. The disease onset is between the age of 15 and 25 years. Patients have symmetrical weakness of the lower limb muscles. Progressive weakness and atrophy of the distal parts of the lower limbs muscles result in foot drop, pes equinovarus deformity and steppage gait. Wasting of upper limbs muscles cause clawing of the hands several years later. Depressed or absent deep tendon reflexes are observed at an early stage of the disease. Mild to moderate sensory impairments including deficits of pain and temperature occur in the feet and hands in all the patients. Fasciculations in the lower and upper limb muscles are observed in elderly patients. Trophic alterations at the feet and knees are an invariant component of the full clinical picture. Cranial nerves are not involved. Intellect and reproductive fitness are conserved and life span is not restricted. The course of the disease is slowly progressive. Disability comes after 15-20 years of evolution although some patients remain fully ambulant after 30 years of disease duration.

Motor median nerve conduction velocities (NCVs) in patients fall within a range of $42.4-58.5 \mathrm{~m} / \mathrm{s}$, with a mean of $47.7 \mathrm{~m} / \mathrm{s}$. For some patients follow up data of consecutive electromyography (EMG) investigations during the last 17 years are available. In these cases a slight decrease of NCVs with age was observed. Compound muscle action potentials (CMAPs) were usually decreased to $30-50 \%$ of normal (to $1.5-2.5 \mathrm{mV}$ ) in hand muscles and to $10 \%$ in feet muscles where the responses were often unrecordable. EMG shows denervation activity and large amplitude and duration of motor units potentials in affected muscles. In conclusion, these electrophysiological findings are characteristic for axonal neuropathy and support the diagnosis of CMT2.

\section{Genetic linkage analysis}

A genome-wide scan was carried out using a fluorescent Human Gene Mapping kit (Weber set, version 6, Isogen Bioscience BV, Maarssen, The Netherlands) with a mean spacing of $10.52 \mathrm{~cm}$. Genotyping was performed on an automated DNA sequencer $\mathrm{ABI} 377$, and the primary data was collected and analysed using the ABI GENESCAN (version 2.0.2) and GENOTYPER (version 2.0) software (Perkin Elmer-Applied Biosystems, Foster-City, CA, USA). Two-point linkage analysis was performed with the LINKAGE package, version 5.1, multipoint linkage was computed with the FASTLINK and VITESSE programms.

\section{Results}

We described a single large six-generation family with autosomal dominant CMT2 (Figure 1). DNA samples from 28 family members including 12 patients were available for genetic linkage analyses. Initially, the CMT1A, CMT1B, CMT2A, CMT2B, CMT2D and CMT2E loci were excluded using a previously described set of short tandem repeat (STR) markers from these regions. ${ }^{3}$ Additionally, the EGR2 gene was excluded using a tightly linked marker, D10S1566. The $\mathrm{X}$-linked CMT loci were not tested because several male-tomale transmissions were present in the family. A genomewide search was performed and genetic linkage to STR markers D7S1818, D7S2204 and D7S2212 on the long arm of chromosome 7 was found. Genetic fine mapping was performed with STR markers D7S2435, D7S669, D7S1797, D7S634, D7S802 and D7S806. In the genetic linkage study, all family members were (based on their current age) allocated to one of six penetrance classes: $0-17,17-19,19-21,21-23$, 23-26 and more than 26 years, with the corresponding average penetrance of $0.08,0.26,0.5,0.74,0.91$ and 0.98 , respectively. The disease frequency was set at 0.0001 . Equal male and female recombination rates were assumed. Allele frequencies for D7S2435 and D7S806 were retrieved from Genome Database (GDB), for other markers alleles were assumed equally frequent. Recombination frequencies between markers were calculated according to the Marshfield Chromosome 7 Sex-Averaged Linkage map (GDB). A maximum two-point LOD score of 5.87 was obtained for D7S2204 (Table 1). Multipoint linkage analysis with markers D7S2435D7S2204-D7S806 resulted in a maximum LOD score of 6.43 at D7S2204 (Figure 2).

\section{Discussion}

We studied a large Russian family with autosomal dominant CMT2 and obtained conclusive linkage to chromosome 7q11-q21. The linkage results and haplotyping data (Figure 1) indicate that the disease gene is located in a $14.5 \mathrm{~cm}$ interval, between D7S2435 and D7S806 as defined by informative recombinants in patients (V.5 and V.8). However, we could further speculate on the localisation of the gene within this interval if we consider recombinants in unaffected individuals. The healthy person III.4 (currently 76 years old) shows for each of the markers D7S634, D7S1797, D7S802, D7S806 and D7S2212 an allele that is also present in linked haplotype. Although the exact haplotype in this person is unknown, we can assume that these alleles actually represent part of the disease haplotype. Moreover, we have tested one additional marker between D7S634 and D7S2212 and six markers telomeric of D7S2212, and again observed the linked alleles further supporting this assumption (data not shown). The probability that III.4 is carrying 12 linked alleles by chance is extremely neglectable. The exact centromeric limit of the disease haplotype in this person could not be determined, since the paternal origin of each individual allele could not be identified. Nevertheless, together these findings indicated that the presence of the gene in the telomeric part of the linked region is unlikely.

So far nine genes involved in CMT and CMT-related neuropathies have been identified. Mutations in PMP22, $M P Z, G J B 1$ and EGR2 are mainly responsible for dominant CMT1 (reviewed in ${ }^{5}$ ), while mutations in NDRG1, MTMR-2 and $P R X$ cause recessive demyelinating neuropathies. ${ }^{6-9}$ 
Table 1 Two-point linkage results with markers from $7 q 11-q 21$ in the CMT2 family from Voronezh province

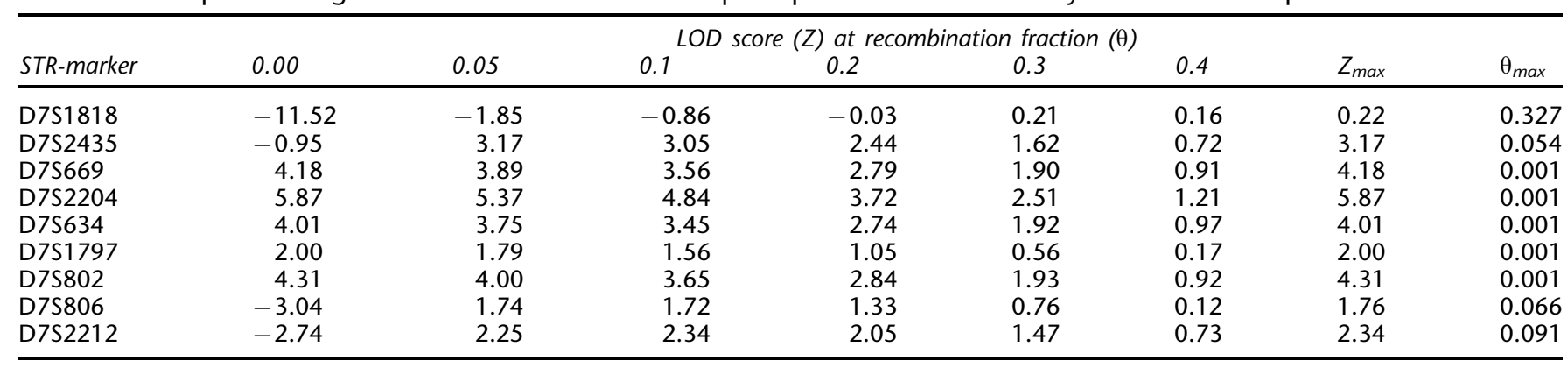

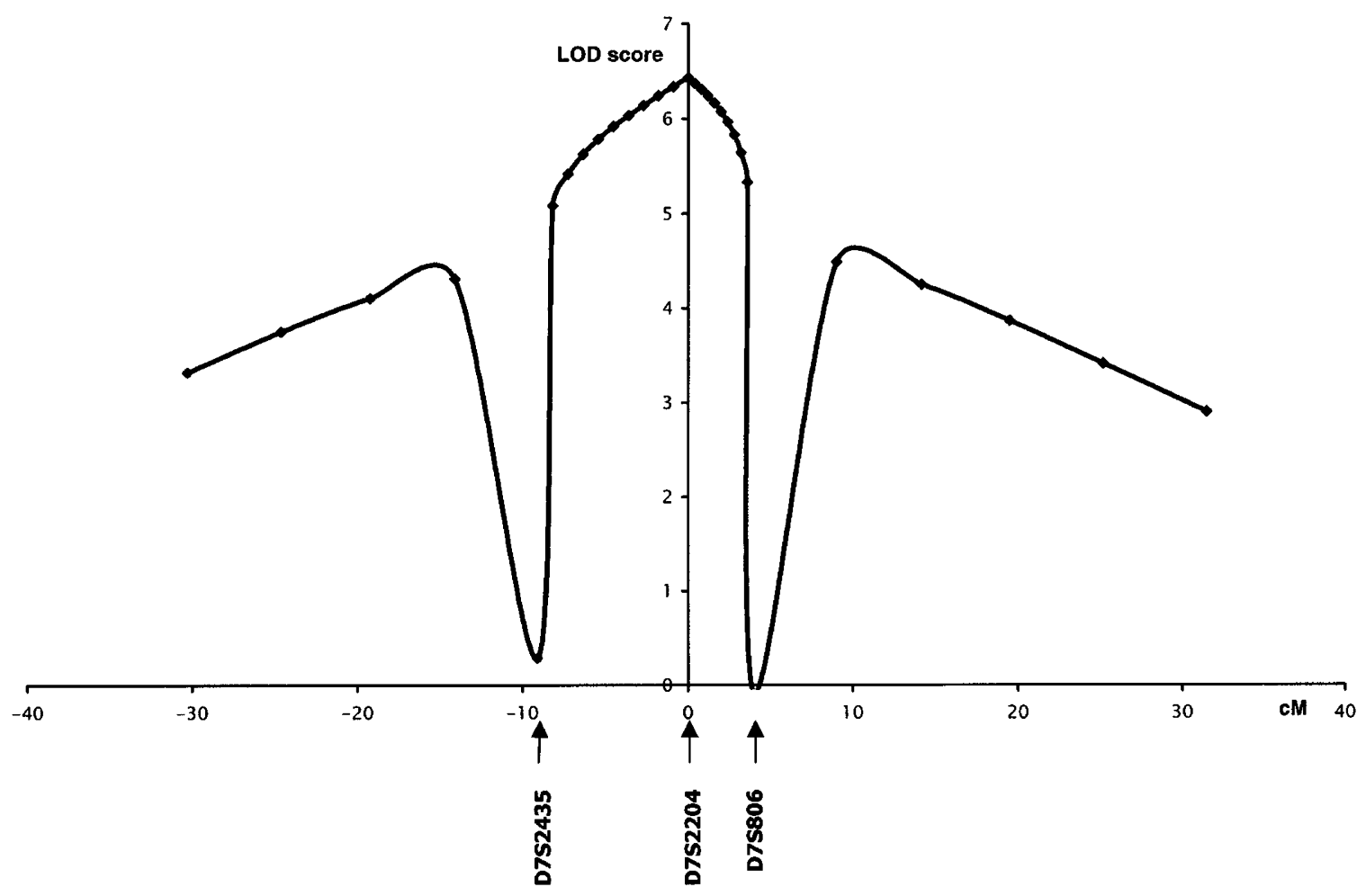

Figure 2 Multipoint linkage analysis at markers D7S2435-D7S2204-D7S806. The maximum LOD score of 6.43 is reached at D7S2204. The genetic distances between the markers are in $\mathrm{CM}$.

Only recently mutations leading to CMT2 have been identified in the gene coding for neurofilament light chain $(N E F L){ }^{3,4}$ while mutations in the cytoskeleton protein gigaxonin (GAN) lead to giant axonal neuropathy. ${ }^{10}$ In most cases, a strict correlation is observed between mutations in a specific gene and the corresponding CMT phenotype. However in rare cases, mutations in genes mainly involved in CMT1 (MPZ and GJB1) can result in a peripheral neuropathy, which officially should be diagnosed as CMT2 based on NCV criteria (reviewed in ${ }^{2}$ and ${ }^{5}$ ).

Up to now 25 loci have been mapped for different forms of CMT and related peripheral neuropathies. Four of these are associated with autosomal dominant CMT2 types; ie CMT2A (MIM 118210) at 1p35-p36; CMT2B (MIM 600882) at 3q13q22; CMT2D (MIM 601472) at 7p14 and CMT2E (MIM $162280)$ at $8 \mathrm{p} 21$. There is also a description of one large pedigree with CMT2 associated with paralysis of diaphragm and vocal cords designated CMT2C. This type of CMT2 has not yet been assigned to a particular chromosome. In addition some other large families with autosomal dominant CMT2 do not show linkage with known CMT2 loci suggesting further genetic heterogeneity (reviewed in ${ }^{2}$ ). In this study, we identified a novel locus for axonal CMT that maps to 7q11-q21, and propose to designate this CMT2 locus 
'CMT2F'. No obvious candidate gene for CMT2F could be identified in the candidate region flanked by D7S2435 and D7S806 localised at 7q11-q21.

\section{Acknowledgments}

The authors wish to thank the patients and their family members for their kind cooperation in this study, and are grateful to Mrs Ann Löfgren, Els De Vriendt and Veerle Van Gerwen for their excellent technical assistance. This research was funded and supported by the Russian Human Genome Organisation (grant No 85), the Fund for Scientific Research (FWO-Flanders, Belgium), the Geneeskundige Stichting Koningin Elisabeth (GSKE, Belgium), the Association Belge contres les Maladies Neuro-musculaires (ABMM, Belgium), the Association Française contre les Myopathies (AFM, France) and the EU-BIOMED2 concerted action of the European CMT consortium (grants CT960055 and CT961614). OV Evgrafov is holder of a research fellowship of the Belgian Office for Scientific, Technical and Cultural Affairs (DWTC, Belgium). V Timmerman is a postdoctoral fellow of the FWO, Belgium.

\section{References}

1 Dyck PJ, Chance P, Lebo R, Carney JA: Hereditary motor and sensory neuropathies. in Dyck PJ, Thomas PK, Griffin JW, Low PA, Poduslo JF (eds). Peripheral Neuropathy. 3 Ed. Philadelphia: W.B. Saunders Company, 1993: pp 1094-1136.
2 De Jonghe P, Timmerman V, Nelis E: Hereditary Peripheral Neuropathies. in: Deymeer F (ed). Neuromuscular Diseases: From Basic Mechanisms to Clinical Management. Basel: Karger, 2000: pp 128-146.

3 Mersiyanova IV, Perepelov AV, Polyakov AV et al: A new variant of Charcot-Marie-Tooth disease type 2 (CMT2E) is probably the result of a mutation in the neurofilament light gene. Am J Hum Genet 2000; 67: $37-46$

4 De Jonghe P, Mersiyanova IV, Nelis E et al: Further evidence that neurofilament light chain gene mutations can cause CharcotMarie-Tooth disease type 2E. Ann Neurol 2001; 49: 245-249

5 Nelis E, Haites N, Van Broeckhoven C: Mutations in the peripheral myelin genes and associated genes in inherited peripheral neuropathies. Hum Mutat 1999; 13: 11-28.

6 Kalaydjieva L, Gresham D, Gooding R et al: N-myc downstreamregulated gene 1 is mutated in hereditary motor and sensory neuropathy-Lom. Am J Hum Genet 2000; 67: 47-58.

7 Bolino A, Muglia M, Conforti FL et al: Charcot-marie-tooth type $4 \mathrm{~B}$ is caused by mutations in the gene encoding myotubularinrelated protein-2. Nat Genet 2000; 25: 17-19.

8 Boerkoel CF, Takashima H, Stankiewicz P et al: Periaxin mutations causes recessive Dejerine-Sottas Neuropathy. Am J Hum Genet 2001; 68: 325-333.

9 Guilbot A, Williams A, Ravisé N et al: A mutation in periaxin is responsible for CMT4F, an autosomal recessive form of CharcotMarie-Tooth disease. Am J Hum Genet 2001; 10: 415-421.

10 Bomont $\mathrm{P}$, Cavalier L, Blondeau $\mathrm{F}$ et al: The gene mutated in giant axonal neuropathy encodes for gigaxonin, a novel member of the cytoskeletal BTB/Kelch repeat family. NatGenet 2000; 26: 370-374. 